\title{
Investigating Darcy-scale assumptions by means of a multiphysics algorithm
}

\author{
Pavel Tomin (pavel.tomin@unil.ch), Ivan Lunati (ivan.lunati@unil.ch) \\ Institute of Earth Sciences (ISTE), University of Lausanne, Switzerland
}

\begin{abstract}
Multiphysics (or hybrid) algorithms, which couple Darcy and pore-scale descriptions of flow through porous media in a single numerical framework, are usually employed to decrease the computational cost of full porescale simulations or to increase the accuracy of pure Darcy-scale simulations when a simple macroscopic description breaks down. Despite the massive increase in available computational power, the application of these techniques remains limited to core-size problems and upscaling remains crucial for practical large-scale applications. In this context, the Hybrid Multiscale Finite Volume (HMsFV) method, which constructs the macroscopic (Darcy-scale) problem directly by numerical averaging of pore-scale flow, offers not only a flexible framework to efficiently deal with multiphysics problems, but also a tool to investigate the assumptions used to derive macroscopic models and to better understand the relationship between pore-scale quantities and the corresponding macroscale variables. Indeed, by direct comparison of the multiphysics solution with a reference pore-scale simulation, we can assess the validity of the closure assumptions inherent to the multiphysics algorithm and infer the consequences for macroscopic models at the Darcy scale. We show that the definition of the scale ratio based on the geometric properties of the porous medium is well justified only for single-phase flow, whereas in case of unstable multiphase flow the nonlinear interplay between different forces creates complex fluid patterns characterized by new spatial scales, which emerge dynamically and weaken the scale-separation assumption. In general, the multiphysics solution proves very robust even when the characteristic size of the fluid-distribution patterns is comparable with the observation length, provided that all relevant physical processes affecting the fluid distribution are considered. This suggests that macroscopic constitutive relationships (e.g., the relative permeability) should account for the fact that they depend not only on the saturation but also on the actual characteristics of the fluid distribution.
\end{abstract}

Keywords: Darcy's law, multiphysics, hybrid, numerical averaging, macroscopic models, multiphase flow

\section{Introduction}

Flow and transport through porous media are determined by the structure of the solid matrix, which is usually assumed inaccessible to the fluids and defines a highly discontinuous and complex pore geometry. Although the physical processes that occur in the pore space are determined by the sub-pore dynamics, the

5 only practicable way to address large-scale problems is to employ macroscopic effective continuum approaches (or simply continuum approaches), which describe the evolution of quantities that are defined by averaging over control volumes of an appropriate size. These approaches have proven effective to model many practical problems as encountered in groundwater and energy applications.

A familiar example is the use of Darcy's law to relate the macroscopic volumetric fluxes (per unit 10 cross area) to the macroscopic pressure gradient. This law (experimentally discovered by Darcy (1856) for one-dimensional single-phase flow with no internal source) can be interpreted as a simplistic momentum balance that assumes separation of spatiotemporal scales (i.e., negligible inertia, hence short relaxation time, and control volumes that are large with respect to the relevant pore-scale lengths). Indicating by $a$ the characteristic microscopic length (i.e., the characteristic length scale of the pore-scale process), and by $H$ the 15 observation length (i.e., the typical size of the macroscopic control volume), Darcy's law is expected accurate if $a / H \ll 1$. Under these conditions (and considered that space and time scale are intimately coupled - 


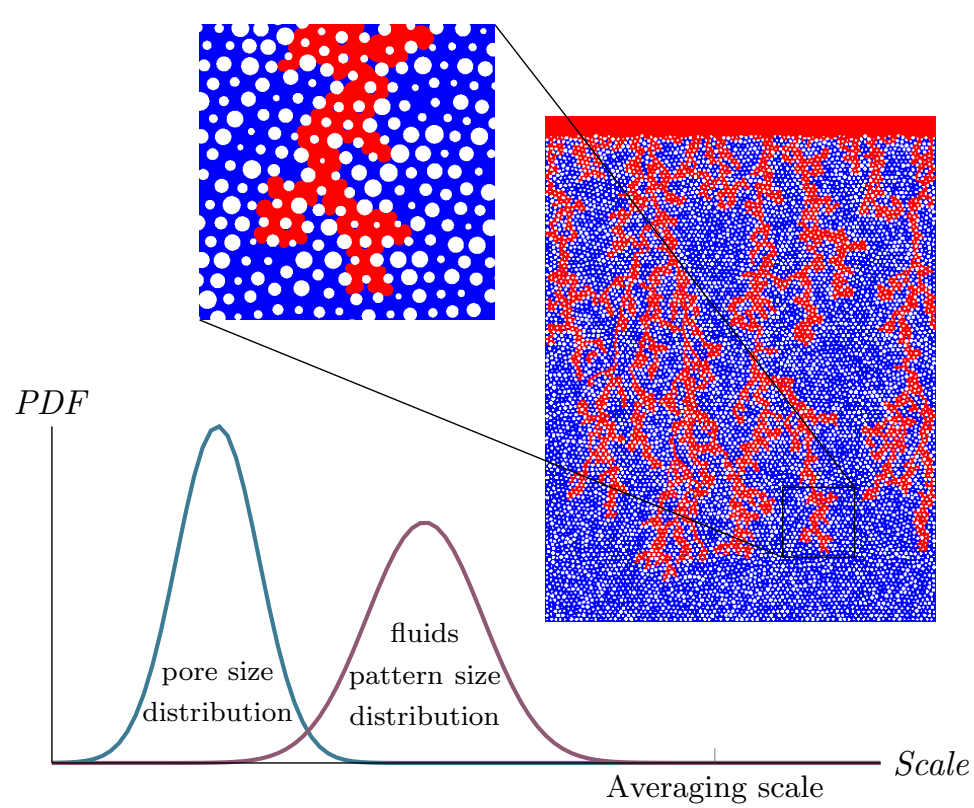

Figure 1: Schematic representation of distribution of spatial scales for multiphase flow through porous media.

see, e.g., Lunati et al. (2002); Tomin and Lunati (2015b)) the drag exerted by the porous matrix within the control volume dominates the viscous fluid-fluid interaction on the external boundary of the control volume and the momentum equation reduces to Darcy's law.

20 In practice, the observation length is generally large compared to the pore size, and scale separation is satisfied in the case of simple (linear) flow phenomena (e.g., transport of an ideal tracer). However, in presence of complex flow, as it is the case when flow and transport are fully coupled, the processes involved are highly nonlinear and unstable regimes might appear (see, e.g., Saffman and Taylor, 1958, Lenormand et al. 1988; Méheust et al., 2002; Løvoll et al., 2004, Kneafsey and Pruess, 2010; Künze and Lunati, 2012; Künze et al. 2014; Ferrari et al., 2015). The system organizes itself into velocity and fluid-distribution patterns that may have a typical size much larger than the original microscopic length scale. When the microscopic and the macroscopic length scales are comparable $(a / H \sim 1)$, Darcy's law may become inaccurate. (See Fig. 1 for a schematic illustration of length scales, and Ferrari and Lunati (2013) and Ferrari et al. (2015) for examples of drainage patterns under different flow regimes).

A crude strategy to deal with the lack of scale separation is to resolve the smallest relevant spatial scale. However, this might require solving the Navier-Stokes equations in the pore space to faithfully describe the complex interplay of forces that determines the velocity and the fluid-distribution patterns. Despite the continuous increase in available computational power, full-resolution direct pore-scale simulations of realisticsize problems will remain intractable (Yang et al., 2013, Molins et al., 2014, Scheibe et al., 2015b; Ferrari et al., 2015). To bypass the computational bottleneck, multiphysics (or hybrid) methods can be employed. They combine pore-scale and Darcy-scale models in a single framework, thus allowing a reduction of the computational cost by adopting a pore-scale (or sub-pore scale) resolution only in small portions of the computational domain, whereas a macroscopic description can be employed elsewhere. In general, this methods are applied to single-phase problems, such as reactive transport (Tartakovsky et al., 2008, Battiato et al. 2011; Scheibe et al., 2015a) or inertial flow regimes (Alyaev et al., 2014).

Applying this strategy to multiphase flow problems is substantially more difficult owing to the immiscibility of the phases, which leads to a fluid distribution that is described by a continuum variable at the macroscale (the saturation), but by a discontinuous function at the pore scale (necessary to describe the evolution of the invading front), which requires the solution of highly nonlinear moving interface problems. Recently, we have proposed a multiphysics method for multiphase flow (the Hybrid Multiscale Finite Volume, 
HMsFV, method (Tomin and Lunati, 2013)), which provides a very general framework to couple different scales and physical descriptions (Tomin and Lunati, 2015a), it is adaptive in space and time (see Tomin and Lunati (2015b) for a detailed discussion of spatiotemporal adaptive simulations of drainage-imbibition cycles), and can be applied to virtually any problem involving multiple physical description (including, e.g., 50 reactive transport, inertial flow regimes, and geomechanical coupling).

The HMsFV method partitions the domain into a set of non-overlapping subdomains (coarse cells) in which local pore-scale problems are solved. These local problems communicate through a global Darcy-scale problem, which provides the boundary conditions. The global problem is constructed by numerical averaging of the microscale equations over the coarse cells (which serve as macroscale control volumes). The accuracy

55 of the multiphysics solutions (with respect to brute-force pore-scale simulations) depends on the ability of the global problem to correctly transfer information between the local problems. As the construction of the global problem is a numerical analog of the conceptual derivation of the macroscopic equations, the method can be used not only as a tool to improve computational efficiency, but also to advance our understanding of the effects of pore-scale processes at larger scales and to investigate the assumptions intrinsic to the macroscopic equations.

In the following, we first describe the HMsFV method focusing on the features that make it an interesting tool to study macroscale models (Sec. 2). Then, we present several numerical tests that aim at investigating the effects of scale separation and of pore-scale physics on the quality of the multiphysics solution (Sec. 3 ). Finally, we conclude by discussing what can be learned from the numerical results about the validity of the

65 Darcy-scale assumptions used to derive the continuum models (Sec. 4).

\section{Multiscale Finite Volume Method}

In the last decade, several multiscale methods have been introduced to incorporate the description of different relevant scales into a single framework (Aarnes et al., 2007, Efendiev and Hou, 2008, Aarnes et al. 2009, Efendiev and Hou, 2009). All these methods aim at reducing the computational costs of solving large 70 elliptic problems by subdividing the original problem into a set of local fine-scale problems coupled through a global coarse problem. As the global problem is constructed by a numerical upscaling procedure, these methods allow an adaptive update of the coarse-scale parameters and permit a more accurate representation of coarse-scale effects of fine-scale dynamics.

Among these methods, the Multiscale Finite Volume (MsFV) method (Jenny et al., 2003) has the peculiar 75 characteristic of building the global problem by averaging the fine-scale conservation equations over auxiliary coarse-scale control volumes. This procedure closely mimics averaging approaches that are used to derive the macroscopic models. By construction, it ensures the consistency between coarse- and fine-scale fluxes and allows recovering an approximate but fully conservative fine-scale solution by solving a second set of local problems (Lunati and Jenny, 2006; Lunati and Lee, 2009). The MsFV method has been extended 80 and applied to a variety of complex problems (Lunati and Jenny, 2006; Wolfsteiner et al. 2006; Lunati and Jenny, 2008; Lee et al., 2008, Hajibeygi and Jenny, 2009, Jenny and Lunati, 2009; Bonfigli and Jenny, 2009. Lunati et al., 2011; Hajibeygi et al., 2011, 2012, Hajibeygi and Tchelepi, 2014, Sandve et al., 2014), but it remained essentially a method to efficiently solve the pressure equation that expresses the conservation of total mass.

Recently, we have presented a Hybrid Multiscale Finite Volume method that couples a pore-scale description at the fine scale with a Darcy-like description at the coarse scale (Tomin and Lunati, 2013), and proposed a reformulation of the method that is adaptive in space and time and can be regarded as a general framework to couple different scales and physical descriptions (Tomin and Lunati, 2015a b). This approach offers the following advantages:

- it is easy to employ different physical descriptions (or different numerical schemes) at the coarse and fine scales (or in different regions of the domain, i.e., for different local fine-scale problems);

- the time steps employed to solve the coarse and the fine problems can be different, in accordance with the fact that fine-scale properties vary more rapidly than coarse-scale properties; 


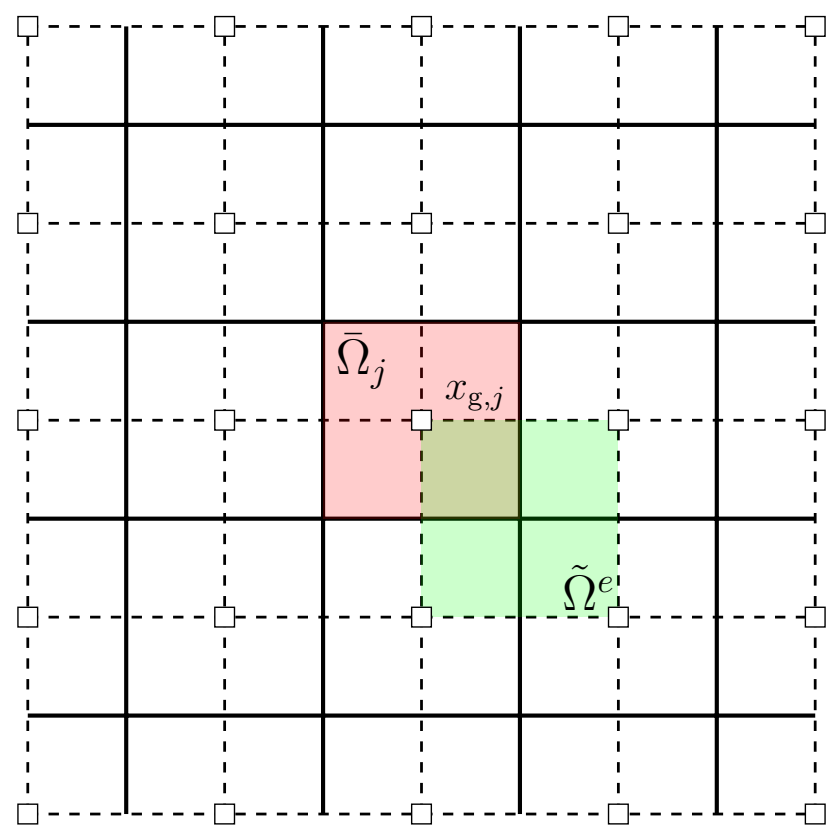

Figure 2: MsFV coarse (solid) and dual (dashed) grids. (The fine-scale grid is not shown here.) The coarse grid is a regular Cartesian grid which partitions the domain into a set of non-overlapping control volumes (usually called "coarse cells" in the MsFV literature). The dual grid is constructed by connecting the centers of the coarse cells, which become the nodes of the dual grid (white squares) and represent the location at which the global degrees of freedom, $x_{\mathrm{g}}$, are defined. Notice that coarse degrees of freedom are also assigned at the domain boundaries.

- on a more technical side, information from the local solutions can be used to improve the quality of the multiscale solution (see Tomin and Lunati (2015a) for a detailed description of this self-correction strategy).

By numerically averaging the micro-scale equations on macro-scale control volumes, the method mimics the derivation of the macroscopic equations and can be used to investigate under which conditions the underlying hypotheses are justified. In the following we briefly describe the components of the algorithm focusing on the properties that are relevant for the numerical averaging.

\subsection{Equations governing multiphase flow at the pore scale}

At the pore scale, we consider the flow of two incompressible phases through a rigid porous medium, which is governed by a system of balance equations for mass and momentum. We use the Volume Of Fluid (VOF) approach (Hirt and Nichols, 1981; Lafaurie et al., 1994, Scardovelli and Zaleski, 1999), which employs a whole-domain formulation and models the two phases as a single fluid with variable properties. The phase distribution is described by the fluid function, $\alpha$, which quantifies the volumetric fraction of one phase. In the following, we assume that $\alpha$ denotes the volume fraction of the nonwetting phase. Therefore, $\alpha=1$ in the region occupied by the nonwetting fluid and $\alpha=0$ in the region occupied by the wetting fluid. Density, $\rho$, and viscosity, $\mu$, vary in space depending on the phase that is locally present, i.e. $\rho(\alpha)=\alpha \rho_{\mathrm{nw}}+(1-\alpha) \rho_{\mathrm{w}}$ and $\mu(\alpha)=\alpha \mu_{\mathrm{nw}}+(1-\alpha) \mu_{\mathrm{w}}$, where $\rho_{\beta}$ and $\mu_{\beta}(\beta=\mathrm{nw}, \mathrm{w})$ are the constant densities and viscosities of the fluids.

As the phases are incompressible, the total mass balance reduces to a divergence-free condition on the velocity, $\boldsymbol{u}$, i.e.

$$
\nabla \cdot \boldsymbol{u}=0
$$

which expresses the conservation of the total volume that can be filled by the fluid phases. 
The momentum balance is given by the Navier-Stokes equations,

$$
\frac{\partial \rho(\alpha) \boldsymbol{u}}{\partial t}+\nabla \cdot(\rho(\alpha) \boldsymbol{u} \boldsymbol{u})=-\nabla p+\nabla \cdot\left[\mu(\alpha)\left(\nabla \boldsymbol{u}+\nabla \boldsymbol{u}^{T}\right)\right]+\gamma \kappa \nabla \alpha,
$$

where $p$ is the pressure, $\gamma$ the surface tension, and $\kappa=\nabla \cdot \boldsymbol{n}_{\alpha}=\nabla \cdot(\nabla \alpha /|\nabla \alpha|)$ the curvature of the fluid-fluid interface ( $\boldsymbol{n}_{\alpha}$ is the unit normal to the fluid-fluid interface). As we are interested in flow at low Reynolds numbers, we neglect the term non-linear in the velocity, but we keep the time derivative because two-phase flow is essentially time-dependent (see Eckhardt and Buehrle (2008) for a discussion).

Finally, the mass balance of the nonwetting phase has the form

$$
\frac{\partial \alpha}{\partial t}+\nabla \cdot(\alpha \boldsymbol{u})=0
$$

The interaction with the solid matrix is described by assigning the no-slip condition for the velocity at the solid boundary $\partial \Omega_{s},\left.\boldsymbol{u}\right|_{\partial \Omega_{s}}=\mathbf{0}$, and prescribing the normal to the fluid-fluid interface

$$
\left.\boldsymbol{n}_{\alpha}\right|_{\partial \Omega_{s}}=\boldsymbol{n}_{s} \cos \theta+\boldsymbol{t}_{s} \sin \theta,
$$

in order to satisfy the equilibrium contact angle, $\theta$, and model the wetting property of the porous medium $\left(\boldsymbol{n}_{s}\right.$ and $\boldsymbol{t}_{s}$ are the unit normal and the unit tangent to $\partial \Omega_{s}$, respectively).

The VOF has been used to model relevant phenomena such as wetting and drying (Afkhami et al., 2009, Lunati and Or, 2009), transition from stable displacement to viscous fingering during drainage (Ferrari and Lunati| 2013), and the roles of inertial effects in controlling the pore-scale front morphology during imbibition (Ferrari and Lunati, 2014). Ferrari et al. (2015) also validated the model by demonstrating that it is capable to provide an accurate description of unstable drainage experiments even by employing relatively unrefined computational grids.

All pore-scale simulations presented here are performed with a modified version of the open-source package OpenFOAM (OpenFOAM, 2012). The computational domain is meshed with the snappyHexMesh grid generator, which is included in the standard OpenFOAM distribution. For more details about the modifications introduced in the original distribution and about the construction of the mesh we refer to (Ferrari and Lunati, 2013; Ferrari et al., 2015).

\subsection{Numerical averaging of the total-mass balance}

The global-scale problem is obtained by averaging the pore-scale equations. To this end, we employ a coarse grid that partitions the entire domain into a set of non-overlapping control volumes (see Fig. 22). (Notice that these control volumes are usually called "coarse cells" in the MsFV literature; here we will use "control volumes" to emphasize their role in constructing coarse-scale balance equations.)

The global total-mass balance of the control volume $\bar{\Omega}_{i}$ is

$$
\int_{\bar{\Omega}_{i}} \nabla \cdot \boldsymbol{u} d \omega=\oint_{\partial \bar{\Omega}_{i}} \boldsymbol{u} \cdot \boldsymbol{n} d a=0
$$

where $\boldsymbol{n}$ is the normal (pointing outwards) to the boundary of the control volume, $\partial \bar{\Omega}_{i}$. (Notice that we use a continuum notation for fine-scale problems and solutions; however, they are solved numerically and the integrals have to be interpreted as summations of cell or face values).

Eq. 5 requires the knowledge of the fluxes across $\partial \bar{\Omega}_{i}$. To make the problem solvable, the integral flux across the boundary needs to be split into a number of macroscopic coarse fluxes, which depend on the coarse-scale unknowns (one for each control volume). This is done with the help of a dual grid, which divides the domain into a second set of subdomains (or dual cells $\tilde{\Omega}^{e}$ ) nonconforming to the previously defined control volumes, such that

$$
\oint_{\partial \bar{\Omega}_{i}} \boldsymbol{u} \cdot \boldsymbol{n} d a=\sum_{e} \int_{\partial \bar{\Omega}_{i} \cap \tilde{\Omega}^{e}} \boldsymbol{u} \cdot \boldsymbol{n} d a=\sum_{e} \mathcal{U}_{i e}=0
$$


where $\mathcal{U}_{i e}$ is implicitly defined by the second equality and represents the flux across the portion of the control-volume boundary, $\partial \bar{\Omega}_{i} \cap \tilde{\Omega}^{e}$. The choice of the dual grid determines the connectivity of the global problem and the ability to capture complex flow patterns. Here we use a staggered dual grid as depicted in Fig. 2, which leads to a 9-point stencil. (Other stencils can be obtained by varying the dual grid; for instance, it is straightforward to obtain a 5-point stencil by employing a diamond grid (Møyner and Lie, 2014)).

\subsection{Interpolators and global-scale conservation of the total mass}

In analogy with Darcy's law, which replaces a true momentum balance equation by a local relationship between flux and pressure gradient, the coarse fluxes, $\mathcal{U}_{i e}$, are obtained from a relationship that relates them directly to the pressure difference. This implies that the pressure values at the nodes of the dual grid (here coincident with the centers of the control volumes) are the only degrees of freedom at the global scale. To construct this relationship, we introduce the localization assumption that the coarse fluxes, $\mathcal{U}_{i e}$, are determined by the pressure distribution on the dual-cell boundary, $\partial \tilde{\Omega}_{e}$, which is constructed by simple linear interpolation of the node values. The pressure at the boundary applies a force that determines the velocity field inside the dual cell, thus the fluxes across the segment of the control-volume boundary. Notice that in this procedure, the pressure is not averaged over a volume (an operation that has no clear physical meaning for an intrinsic variable), but a surface averaging is instead applied (pressure multiplied by the surface area represents the force acting on the corresponding surface element, which is additive and can be averaged). This is also closely related to experimental measurement techniques, in which pressure is never measured over a volume but over the sensor surface.

Assigning a unit pressure at each node $j$, we construct a set of basis functions $\left(\phi_{j}^{e}, \boldsymbol{\psi}_{j}^{e}\right)$, which entails scalar basis functions for the pressure, $\phi_{j}^{e}$, and vector basis functions for the velocity, $\boldsymbol{\psi}_{j}^{e}$. To describe processes that do not scale with the global pressure (e.g. capillarity or gravity), we construct a set of correction functions $\left(\phi_{0}^{e}, \boldsymbol{\psi}_{0}^{e}\right)$ by assigning zero pressure at $\partial \tilde{\Omega}^{e}$. (Here, we restrict ourselves to a twodimensional problem; in three dimensions, the procedure described above is first applied to each face of the dual cell, and the resulting pressure distribution is then used as boundary condition to solve a threedimensional problem in the interior of $\tilde{\Omega}_{e}$.)

Basis and correction functions are interpolators that allow us to express pressure and velocity distributions in the dual cells as

$$
\left.p\right|_{\tilde{\Omega}_{e}}=\sum_{j} p_{\mathrm{g}, j} \phi_{j}^{e}+\phi_{0}^{e} \quad \text { and }\left.\quad \boldsymbol{u}\right|_{\tilde{\Omega}_{e}}=\sum_{j} p_{\mathrm{g}, j} \boldsymbol{\psi}_{j}^{e}+\boldsymbol{\psi}_{0}^{e}
$$

respectively, where $p_{\mathrm{g}, j}$ are the unknown global pressures. (Notice that this splitting is possible because the equations are linear in pressure and velocity.) Inserting the interpolated velocity into Eq. 6, we obtain the coarse-scale fluxes

$$
\mathcal{U}_{i e}=\int_{\partial \bar{\Omega}_{i} \cap \tilde{\Omega}^{e}} \boldsymbol{u} \cdot \boldsymbol{n} d a=\int_{\partial \bar{\Omega}_{i} \cap \tilde{\Omega}^{e}}\left(\sum_{j} p_{\mathrm{g}, j} \boldsymbol{\psi}_{j}^{e}+\boldsymbol{\psi}_{0}^{e}\right) \cdot \boldsymbol{n} d a=\sum_{j} t_{i j}^{e} p_{\mathrm{g}, j}-r_{i}^{e},
$$

where we have defined

$$
t_{i j}^{e}=\int_{\partial \bar{\Omega}_{i} \cap \tilde{\Omega}^{e}} \boldsymbol{\psi}_{j}^{e} \cdot \boldsymbol{n} d a \text { and } r_{i}^{e}=-\int_{\partial \bar{\Omega}_{i} \cap \tilde{\Omega}^{e}} \boldsymbol{\psi}_{0}^{e} \cdot \boldsymbol{n} d a .
$$

Eq. 8 is analogous to standard Darcy equation, which also contains additional terms that do not scale with pressure $\left(r_{i}^{e}\right.$ in our case) in presence of capillary or gravity forces.

We can write the total mass conservation equation for the cell $\bar{\Omega}_{i}$ in the form

$$
\sum_{e} \mathcal{U}_{i e}=\sum_{j} \mathcal{T}_{i j} p_{\mathrm{g}, j}-\mathcal{R}_{i}=0
$$


where $\mathcal{T}_{i j}=\sum_{e} t_{i j}^{e}$ are the coarse-scale transmissibilities, and $\mathcal{R}_{i}=\sum_{e} r_{i}^{e}$ is the right-hand side. Using the constraints

$$
\sum_{j} \phi_{j}^{e}=1 \quad \text { and } \quad \sum_{j} \boldsymbol{\psi}_{j}^{e}=\mathbf{0}
$$

to ensure zero fluxes in the case of constant pressure, we can rewrite the mass conservation Eq. 10 in the form

$$
\sum_{j \neq i} \mathcal{T}_{i j}\left(p_{\mathrm{g}, j}-p_{\mathrm{g}, i}\right)=\mathcal{R}_{i}
$$

which is analogous to the discrete mass conservation equation obtained employing Darcy's law.

\subsection{Numerical closure and definition of the interpolators}

The form of the localized problems that define the interpolators (basis and correction functions) determines the physical processes that are taken into account to compute the fluxes across the control volumes and provides a numerical closure of the global problem. Following (Tomin and Lunati, 2013), we start considering the equations that describe the full physics at the pore scale. For basis functions we have

$$
\left\{\begin{array}{l}
\nabla \cdot \boldsymbol{\psi}_{j}^{e}=0, \\
\rho(\alpha) \frac{\partial \boldsymbol{\psi}_{j}^{e}}{\partial t}=-\nabla \phi_{j}^{e}+\nabla \cdot\left[\mu(\alpha)\left(\nabla \boldsymbol{\psi}_{j}^{e}+\nabla \boldsymbol{\psi}_{j}^{e T}\right)\right],
\end{array}\right.
$$

which are solved for each node $j$ of the dual cell $\tilde{\Omega}^{e}$. Dirichlet boundary conditions are imposed for the pressure at $\partial \tilde{\Omega}^{e}: \phi_{j}^{e}=1$ is assigned at the node $j, \phi_{j}^{e}=0$ at the other nodes of the dual, and linear variation is assumed between the nodes. For the velocity, a zero normal-gradient condition is imposed on $\partial \tilde{\Omega}^{e}$, and a zero initial condition is used. The correction functions, $\left(\phi_{0}^{e}, \boldsymbol{\psi}_{0}^{e}\right)$, are obtained by solving analogous problems for each dual, $\tilde{\Omega}^{e}$,

$$
\left\{\begin{array}{l}
\nabla \cdot \boldsymbol{\psi}_{0}^{e}=0, \\
\rho(\alpha) \frac{\partial \boldsymbol{\psi}_{0}^{e}}{\partial t}=-\nabla \phi_{0}^{e}+\nabla \cdot\left[\mu(\alpha)\left(\nabla \boldsymbol{\psi}_{0}^{e}+\nabla \boldsymbol{\psi}_{0}^{e T}\right)\right]+\gamma \kappa \nabla \alpha,
\end{array}\right.
$$

which contain the effects of the surface tension (the last term on the right-hand side). The boundary conditions are similar to Eq. 13 , except that $\phi_{0}^{e}$ is fixed at zero on the whole boundary $\partial \tilde{\Omega}^{e}$. The velocity at previous time is used as initial condition for $\boldsymbol{\psi}_{0}^{e}$. Examples of basis and correction functions are shown in Fig. 3 .

The coarse-scale transmissibilities obtained from the velocity basis functions, $\mathcal{T}_{i j}$, represent the total fluid mobilities and describe the coarse fluxes generated by the pressure differences between the control volumes. The coarse-scale right-hand side, $\mathcal{R}_{i}$, mainly describes the effects of the capillary forces on the macroscopic mass balance (and also includes part of velocity-relaxation effects). Due to the form of the problems that define the interpolators (Eqs. 13 and 14), $\mathcal{T}_{i j}$ and $\mathcal{R}_{i}$ contain important pore-scale information and represent an extension of the standard Darcy model:

1) the interpolators depend on the actual phase distribution in the pores through the dependence of density and viscosity on the pore-scale fluid function (and not simply on the average saturation);

2) the effect of surface tension also depends on the actual phase distribution;

3) pore-scale non-equilibrium effects are described by the presence of the time derivatives, which account for non-zero relaxation time. 
$\phi_{1}$
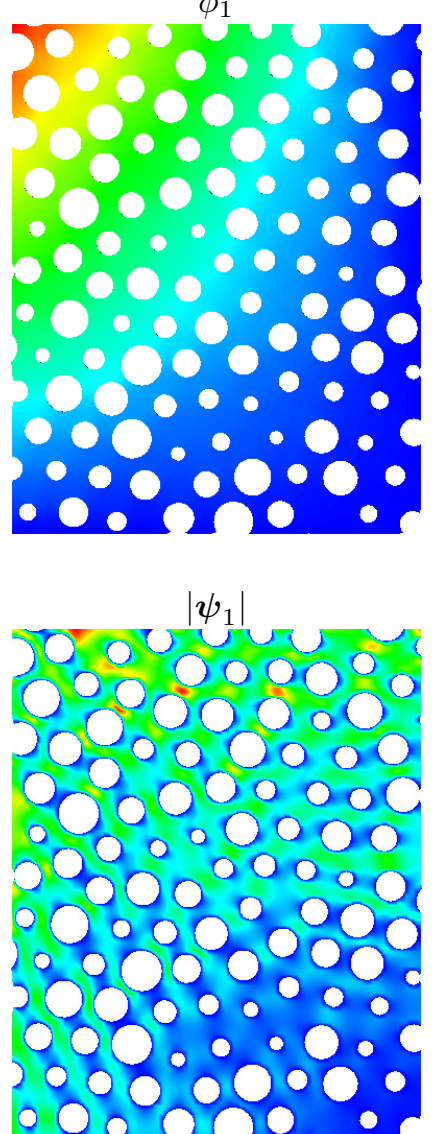

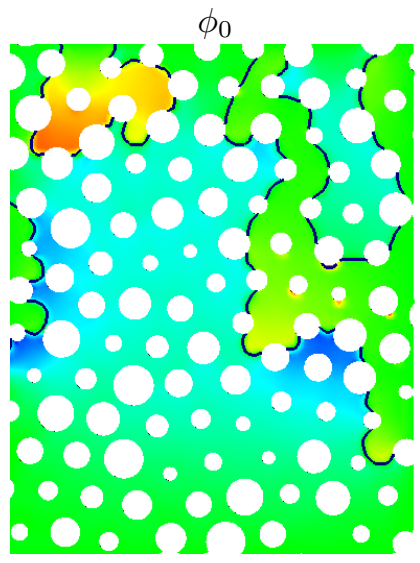

\section{$\left|\boldsymbol{\psi}_{0}\right|$}

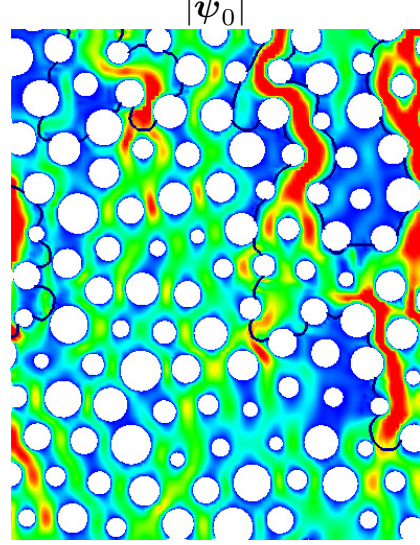

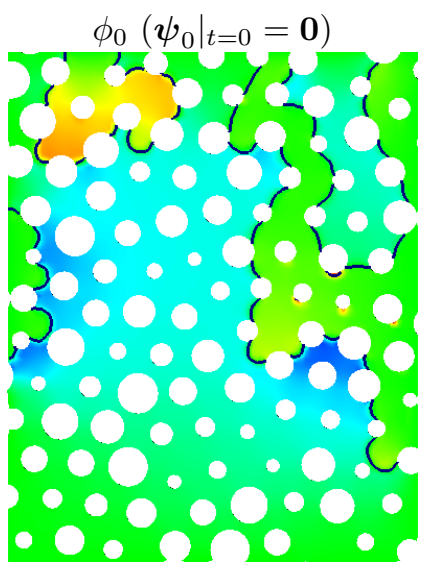

$\left|\boldsymbol{\psi}_{0}\right|\left(\left.\boldsymbol{\psi}_{0}\right|_{t=0}=\mathbf{0}\right)$

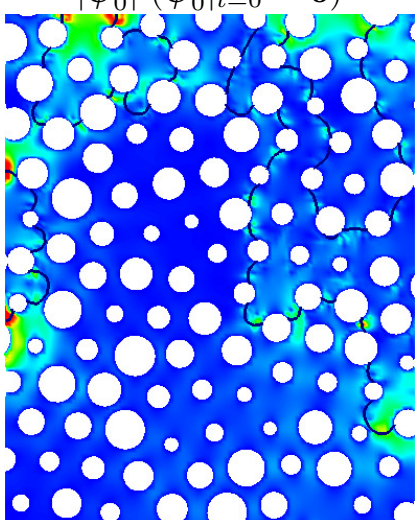

Figure 3: Pressure (top) and velocity (bottom) interpolators. Examples of basis and correction functions are shown in the left and mid columns, respectively; whereas the right column illustrates the contribution of the surface tension to the correction function (in the mid and the right columns, the black lines indicate the position of the fluid-fluid interface). Blue (resp. red) corresponds to low (resp. high) values. Notice that the color scale for basis and correction functions is different, and that only the absolute value of the velocity interpolators is shown. 


\subsection{Solution strategy and hypotheses}

Following the solution strategy outlined in Tomin and Lunati (2015a), we consider a set of local problems defined on control volumes (Fig. 2), for which we solve the full system of equations governing the pore-scale flow, Eqs. 1 13 The boundary conditions for Eqs. 1 and 2 are obtained from the solution of the global equation for the total mass balance (Eq. 12), which is interpolated onto the control-volume boundaries by means of basis and correction functions (Eq. 7). Therefore, the local problems are coupled through the boundary conditions assigned on $\partial \bar{\Omega}_{i}$, which rely on the assumptions introduced by the numerical closure used to compute the interpolators and to construct the global problem (e.g., that the global pressure is the only macroscopic independent variable). Eq. 3 is hyperbolic and it is solved explicitly using the MULES scheme (Bohorquez, 2008, OpenFOAM, 2012); therefore, the local boundary conditions for the fluid function are not constructed by means of interpolation, but are simply taken from the values at the previous time.

The local fine-scale problems on control volumes are solved with smaller time steps than the global coarse problem. This means that the pressure and the velocity boundary conditions of the local problems are not updated at each local time step, but only when the changes in fluid distribution are large enough to affect the total fluid mobility at the coarse-scale. These mobility changes are estimated by monitoring the pressure variation (with respect to the pressure at the previous update) on the boundaries of the control volumes (see Tomin and Lunati (2015a) for details). When the estimated mobility changes are large, the global solution is computed by updating the interpolators (with the new fluid distribution, also new initial condition for the velocity goes to the correction functions), solving the global problem, and updating the boundary conditions of local problems (including the values assigned to $\alpha$ ). The time step of the pore-scale problems on the control volumes is controlled by the Courant-Friedrichs-Lewy (CFL) condition for Eq. 3. which is solved with a time-explicit scheme, and the same time step is used to compute the interpolators.

Mass conservation is guaranteed by the solution of the Eq. 12, by the consistency between fine-scale and coarse-scale fluxes, and by the fact that, at the boundary of the control volumes, the fluid function is updated at each local time step. Momentum conservation is not enforced at the coarse scale. The underlying assumption that the coarse-scale fluxes, $\mathcal{U}_{i e}$, are fully determined by the local pressure imposed on $\partial \tilde{\Omega}^{e}$ corresponds to a Darcy-like relationship between flux and pressure gradient, in which the latter is interpreted as the resultant of the pressure force (per unit volume) applied at the boundary.

In addition to the hypotheses above, the quality of the solution also depends on the level of detail at which the interpolators describe the local physical processes. In the following, we will test the ability of different interpolators to transfer information across the coarse control volumes and to faithfully reproduce the coarse-scale fluxes. We will therefore compare the results obtained by solving the pore-scale governing equations on the whole domain with the results of the multiphysics method. To asses the importance of including the corresponding physical processes in a Darcy-like relationship, we also test interpolators that entail a simplified physical description. Therefore, in addition to the basis and correction functions described in Eqs. 13 and 14, respectively, we will also

- consider interpolators that neglect time derivatives, in order to assess the importance of including relaxation effects in the coarse-scale mobilities, $\mathcal{T}_{i j}$, and in the capillary pressure term, $\mathcal{R}_{i}$;

- neglect the correction functions to show that neglecting relevant physical mechanisms in the fluxes across control volumes may lead to inaccurate solutions even if the full system of pore-scale equations is locally solved on $\bar{\Omega}_{i}$.

\section{Numerical examples}

We consider two pore geometries that are constructed from a two-dimensional packing of grains (circles) obtained by simulating deposition (Vinningland et al., 2007). The first geometry (uncorrelated geometry, Fig. 4a) is obtained by applying a random compression to the sedimented grains (see also Ferrari and Lunati (2013)). The compression allows obtaining a connected pore space of the desired porosity, which has an uncorrelated distribution of the pore size. The second geometry (correlated geometry, Fig. 4ab) is constructed starting with an uncorrelated geometry, and then removing the grains that fall within a set of connected 


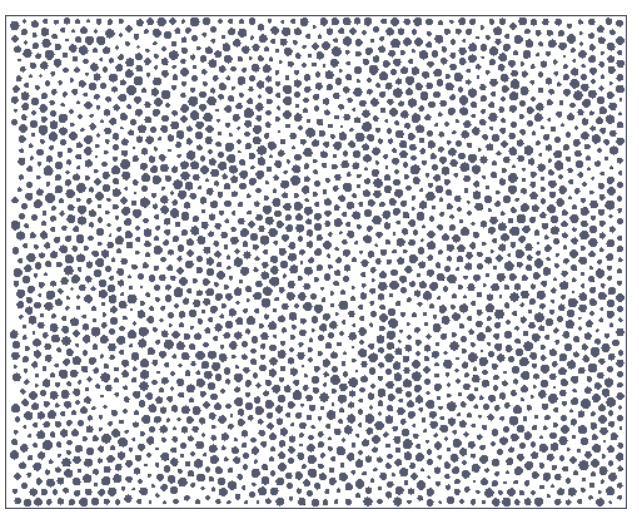

(a) uncorrelated

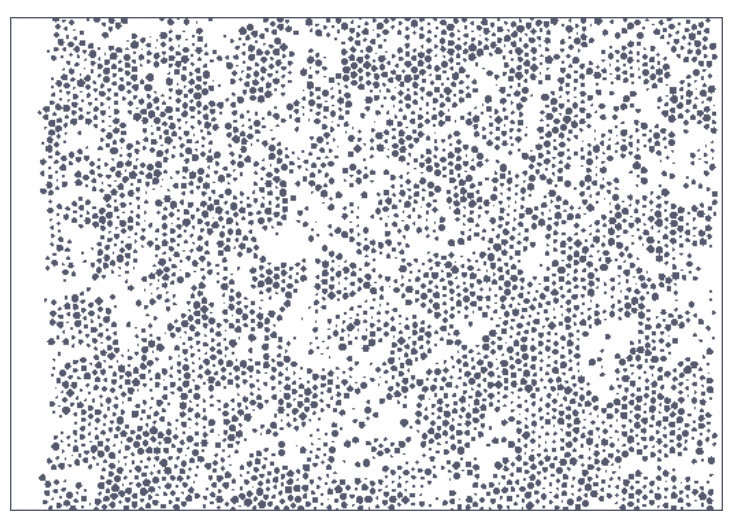

(b) correlated

Figure 4: Pore geometries. The geometries are constructed from a two-dimensional packing of circles by applying a random compression in order to obtain a connected pore space. The correlated geometry is created by additionally removing the circles falling within connected channels.

channels generated by transformations of a Gaussian field (Tyukhova et al., 2015). This procedure allows us to obtain a geometry that is characterized by a larger pore-scale length, taken to be the channel length. The properties of the two geometries are summarized in Tab. 1 .

\subsection{Single-phase flow and the scale-separation hypothesis}

We fist consider a simple single-phase flow problem: a fluid with viscosity $\mu=0.1 \mathrm{~kg} \mathrm{~m}^{-1} \mathrm{~s}^{-1}$ and density $\rho=1000 \mathrm{~kg} \mathrm{~m}^{-3}$ is injected at the left boundary with uniform velocity $v_{\text {in }}=10^{-4} \mathrm{~m} \mathrm{~s}^{-1}$, while the pressure is fixed at the right boundary, $p_{\text {out }}=0$. The reference solutions for both geometries are obtained by solving the problem with a pore-scale solver. The multiphysics solutions are computed for different coarse grids. By varying the coarse grid we modify the ratio between the pore-scale characteristic length, $a$, and the observation length, $H$, represented by the size of the control volumes. Weakening the scale separation condition, $a / H \sim 1$, increases the error of the multiphysics solution with respect to the reference pore-scale simulation. Physically, this arises from an unbalance between coarse pressure and viscous forces, and leads to a larger variance of the velocity (Fig. 5). The reduced pressure force can be interpreted as an overestimation of the effective permeability (the flux is fixed), and the increase of the variance as an overestimation of macroscopic dispersion.

In the correlated geometry, the presence of long connected structures requires larger control volumes to satisfy the scale separation hypothesis. If the problem is linear (as the transport of an ideal tracer) only the static length scales characterizing the medium structure are relevant, but in case of highly nonlinear problems the invasion process may become unstable, promoting the formation of dynamic preferential paths (fingers) and rendering irrelevant the definition of scale separation that is based only on the pore geometry.

\subsection{Two-phase displacement}

In case of two-phase flow, the process may become unstable leading to the situation when fluids organize themselves in patterns that display a transverse size of a few pores and a longitudinal size spanning many

\begin{tabular}{cccccccc}
\hline Geometry & $\begin{array}{c}\text { Domain } \\
\text { size, mm }\end{array}$ & $\begin{array}{c}\text { Mean } \\
\text { obstacle } \\
\text { size, } \mathrm{mm}\end{array}$ & $\begin{array}{c}\text { Mean pore } \\
\text { size, mm }\end{array}$ & $\begin{array}{c}\text { Number of } \\
\text { obstacles }\end{array}$ & $\begin{array}{c}\text { Number } \\
\text { of cells }\end{array}$ & $\begin{array}{c}\text { Permeability, Porosity } \\
\mathrm{m}^{2}\end{array}$ & \\
\hline Uncorrelated & $56.8 \times 45$ & 0.6 & 0.4 & 2507 & 711720 & $10^{-8}$ & 0.70 \\
Correlated & $217 \times 150$ & 0.75 & 3.5 & 4087 & 2464671 & $10^{-8}$ & 0.75 \\
\hline
\end{tabular}

Table 1: Properties of the pore geometries. The permeability is calculated by means of single-phase numerical simulations. 

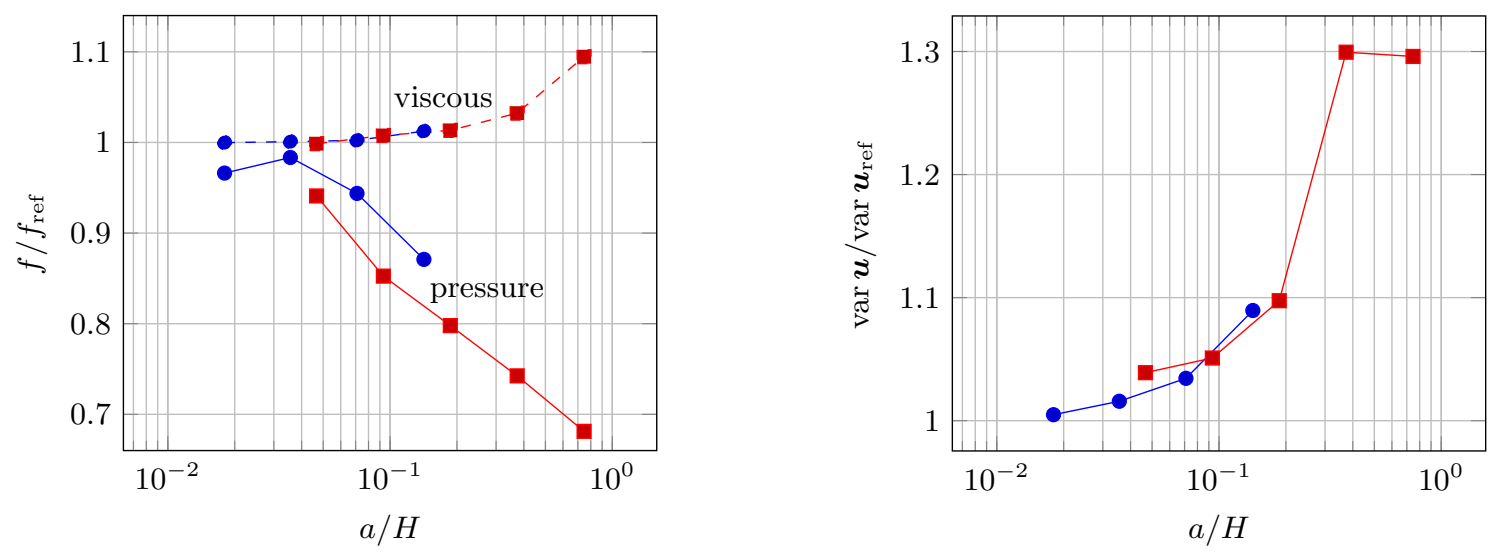

$\longrightarrow$ uncorr. $\rightarrow$ - corr.

Figure 5: Average forces (pressure and viscous) and velocity variance for multiphysics solutions with different scale ratios (coarse resolutions $2 \times 2, \ldots, 32 \times 32$ ) with respect to the fine-scale reference solution.

pores. The pore-scale process is then characterized by the typical size of the fluid patterns that depends on the interplay between capillarity and viscous forces for the specific flow regime and it is much larger than the length that characterizes the pore geometry.

To illustrate the effects of these emerging structures on macroscopic quantities, we consider the uncorrelated geometry (Fig. 4a), which is characterized by a single spatial scale, i.e., the mean pore size. The nonwetting fluid is injected at the left boundary with a constant velocity $v_{\text {in }}=10^{-2} \mathrm{~m} \mathrm{~s}^{-1}$, while the pressure is fixed at the right boundary, $p_{\text {out }}=0$. The properties of the fluids are summarized in Tab. 2 and the capillary number is $C a=\mu_{\mathrm{w}} a^{2} v_{\text {in }} /(k \gamma) \sim 0.1$, where $k$ is the permeability.

First, we consider an unstable drainage that occurs at slightly unfavorable viscosity ratio, $M=\mu_{\mathrm{nw}} / \mu_{\mathrm{w}}=$ 0.2. Similarly to the single-phase results, we observe that local differences in the fluids distribution between the multiphysics and the reference solutions tend to increase when scale separation is weakened (compare, e.g., Fig. 6a and Fig. 6p). Small fluctuations grow with time for unstable regimes, such that an exact pointby-point correspondence between the multiphysics solution and the reference is not expected. If we compare the solutions in a statistic sense by plotting the average longitudinal profiles of the saturation (Fig. 7, left), we observe that all multiphysics solutions are quite close to the reference for any coarse grid. In order to characterize the differences between the flow patterns, we plot the fluid-fluid interfacial area as a function of the pore volume injected (Fig. 7, right). Similarly to the results in Tomin and Lunati (2013), the interfacial area evolution is very well reproduced with the multiphysics algorithm (the maximum error is about $5 \%$ ).

Next, we increase the flow instability by lowering the viscosity ratio to $M=4 \cdot 10^{-4}$. This leads to a fully developed viscous-fingering regime, which is characterized by an invading structure branching into thin fingers. The multiphysics solution sensibly deteriorates when the control volumes become smaller and the scale ratio increases. The average longitudinal profiles of the saturation (Fig. 9, left) show that the multiphysics solution becomes rather inaccurate already when a $4 \times 4$ dual grid is employed (see also

\begin{tabular}{|c|c|}
\hline Interfacial tension, $\gamma$ & $0.064 \mathrm{~kg} \mathrm{~s}^{-2}$ \\
\hline Contact angle, $\theta$ & 30 deg. \\
\hline Wetting fluid density, $\rho_{\mathrm{w}}$ & $800 \mathrm{~kg} \mathrm{~m}^{-3}$ \\
\hline Density ratio, $\rho_{\mathrm{nw}} / \rho_{\mathrm{w}}$ & 1.25 \\
\hline Wetting fluid viscosity, $\mu_{\mathrm{w}}$ & $0.05 \mathrm{~kg} \mathrm{~m}^{-1} \mathrm{~s}^{-1}$ \\
\hline Viscosity ratio, $M=\mu_{\mathrm{nw}} / \mu_{\mathrm{w}}$ & $4 \cdot 10^{-4}$ \\
\hline
\end{tabular}

Table 2: Fluids properties. 


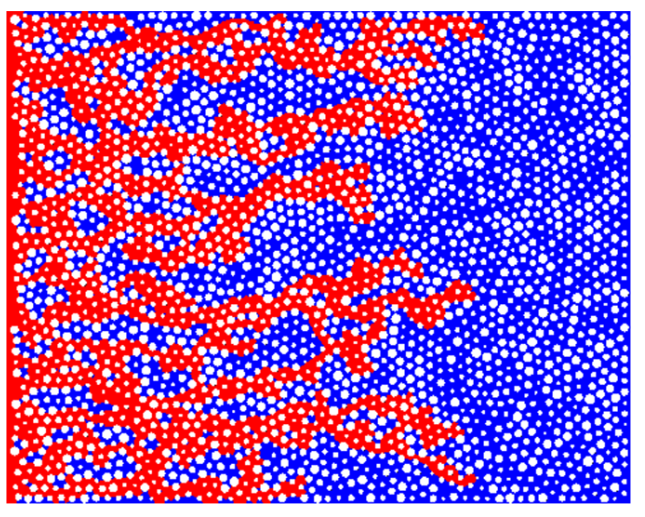

(a) reference

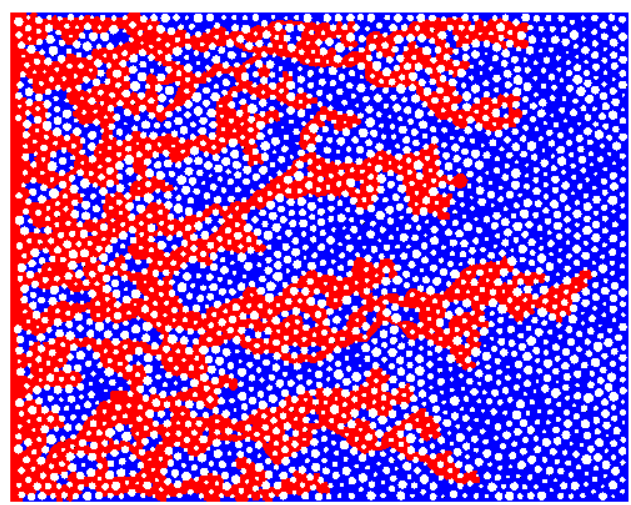

(c) $[\partial f \partial t]_{b}$

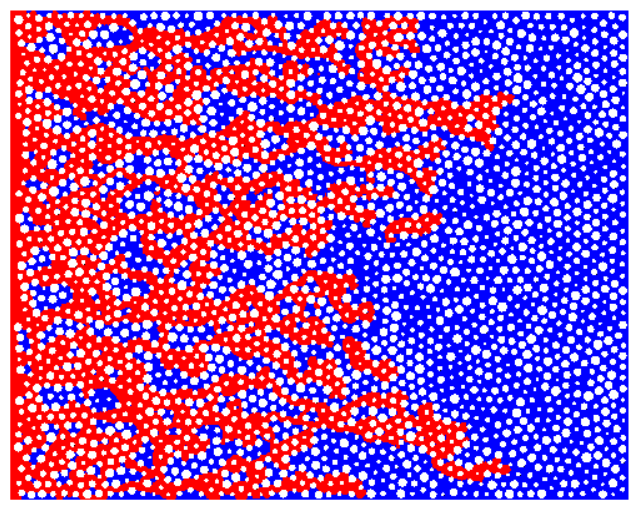

(e) $\left(\phi_{0}^{e}, \phi_{0}^{e}\right)+[\partial f \partial t]_{b}$

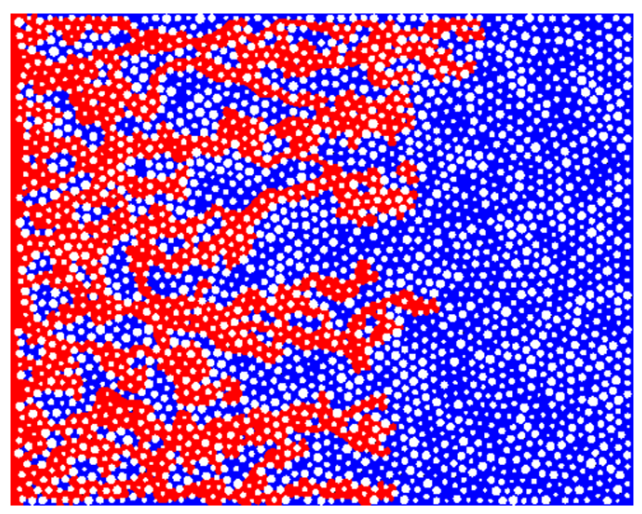

(b) full-physics

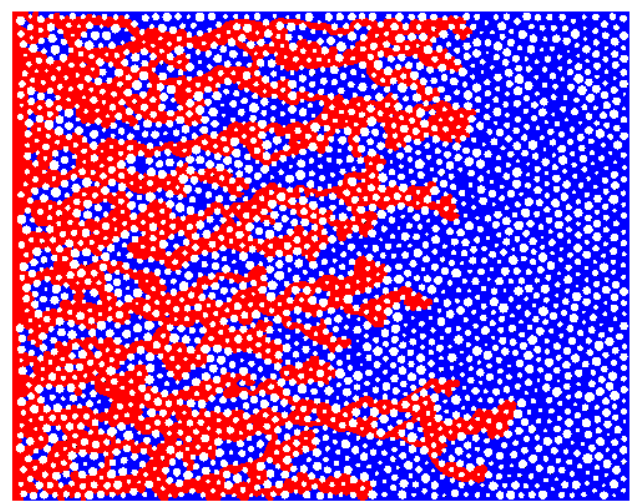

(d) $[\partial f \partial t]_{b+c}$

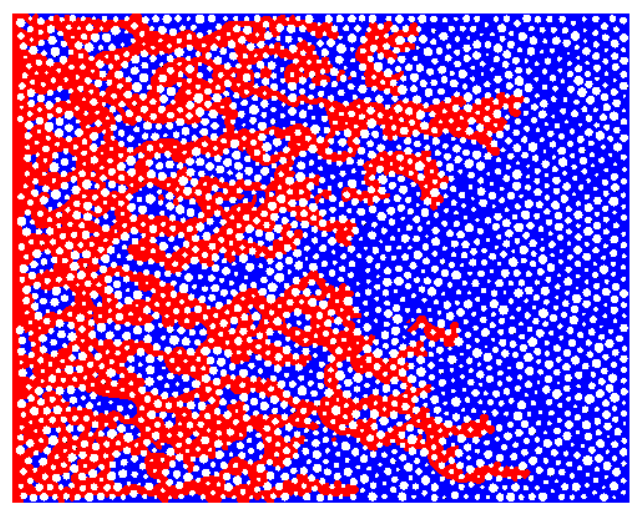

(f) $\not k \nabla \alpha$

Figure 6: Drainage simulations for $M=0.2$ : (a) fine-scale reference solution, (b) multiphysics solution with full-physics interpolators, (c-f) multiphysics solutions with simplified interpolators. (c) no time derivative for basis functions, (d) no time derivative for basis and correction functions, (e) no correction function and no time derivative for basis functions, and (f) no surface tension for correction function. Dual grid has $4 \times 4$ cells. 

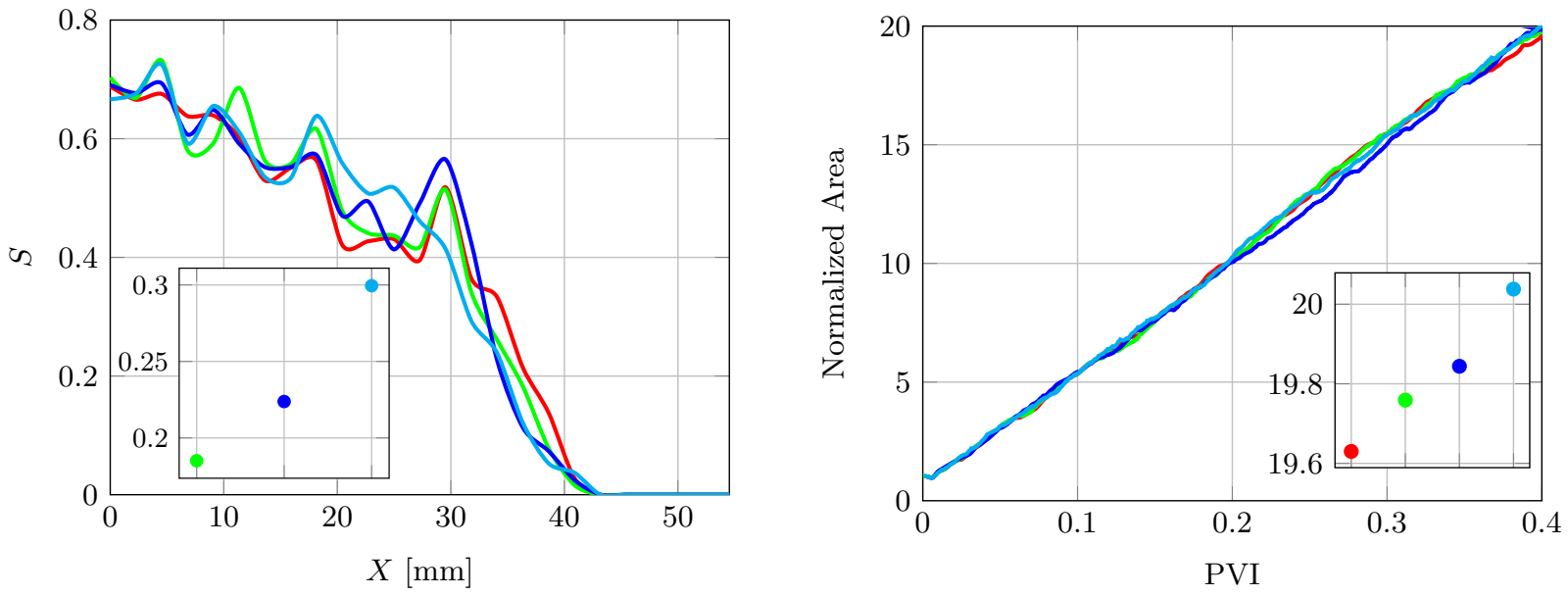

ref $2 \times 2-4 \times 4-8 \times 8$

Figure 7: Left: average longitudinal profiles of the saturation; the subplot shows the $\mathrm{L}_{2}$-norm difference between the multiphysics and reference curves. Right: fluid-fluid interfacial area (normalized by its value at initial condition) as a function of pore volume injected (PVI) (right); the subplot shows the normalized area values at PVI $=0.4$. Viscosity ratio is $M=0.2$.

Fig. $8 \mathrm{a}, \mathrm{b})$. The evolution of the interfacial area with the pore volume injected confirms the deterioration of the solution when the coarse-grid size decreases (Fig. 9, right). The lack of scale separation between the size of the invading structure and the observation scale affects the front morphology, leading to finer fingers and to a substantial overestimation of the fluid-fluid interface (about $14 \%$ larger).

\subsection{Coarse-scale transmissibility}

The coarse scale transmissibility, $\mathcal{T}_{i j}$, relates the macroscopic pressure difference to the macroscopic total flux. it is the analog of the total mobility used in fractional flow formulations for standard Darcy-scale models, but with an important difference. The total mobility is defined as

$$
\lambda=\left(\frac{k_{\mathrm{w}}^{r}}{\mu_{\mathrm{w}}}+\frac{k_{\mathrm{nw}}^{r}}{\mu_{\mathrm{nw}}}\right) k,
$$

where the wetting and nonwetting relative permeabilities (resp. $k_{\mathrm{w}}^{r}$ and $k_{\mathrm{nw}}^{r}$ ) depend only on the average local fluid content, i.e., on the saturation (see, e.g., Aziz and Settari (1979); Bear and Verruijt (1987); Helmig (1997); Holzbecher (1998)). (Notice that this assumption can be partially relaxed if an hysteretic model is introduced). The multiphysics transmissibility, instead, contains the complete information about the detailed fluid distribution in the pore-space. Therefore, it contains much more information and represents an extension of the standard total mobility.

In Fig. 10 we plot the coarse-scale two-phase transmissibilities normalized by the single phase transmissibilities, $\mathcal{T}_{i j}(S) / \mathcal{T}_{i j}(0)$, as a function of the average control-volume saturation for $4 \times 4$ and $8 \times 8$ dual grids. There is a substantial difference between the curves corresponding to the individual control volumes. More important, some curves display a very complex behavior, which cannot be reduced to classical relative mobility models. Despite the small scale ratio between pore and control-volume sizes $(a / H \approx 0.036$ for the $4 \times 4$ grid), the presence of the new length that characterizes the fluid pattern weakens the scale separation hypothesis, rendering the fluid distribution in the control volume not statistically representative. This effect is of course more dramatic in the case of small control volumes (i.e., with the $8 \times 8$ dual grid, see Fig. 10p).

\subsection{Coarse-scale effects of time derivative and capillarity}

As we have discussed in the previous section, the coarse-scale transmissibilites are able to describe a variety of flow regimes owing to the fact that they contain full information about the morphology of the 


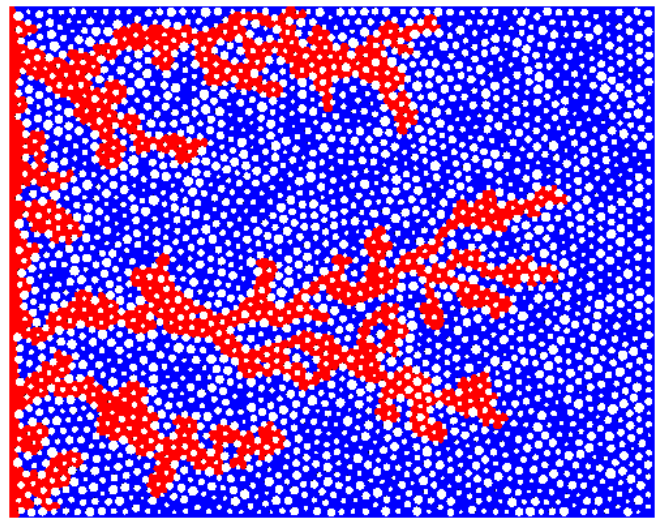

(a) reference

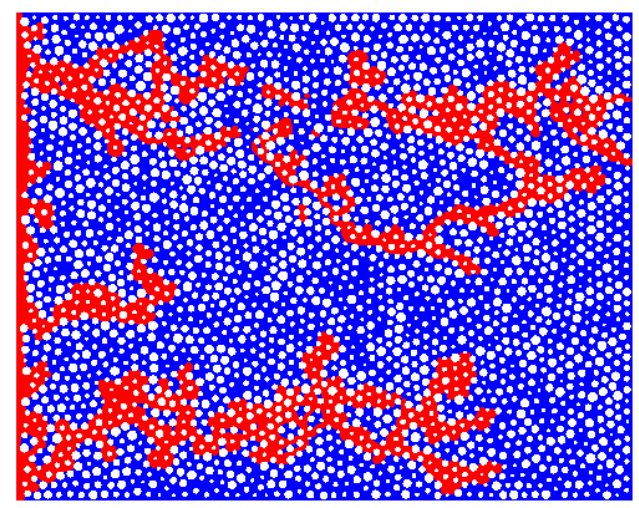

(c) $[\partial f \partial t]_{b}$

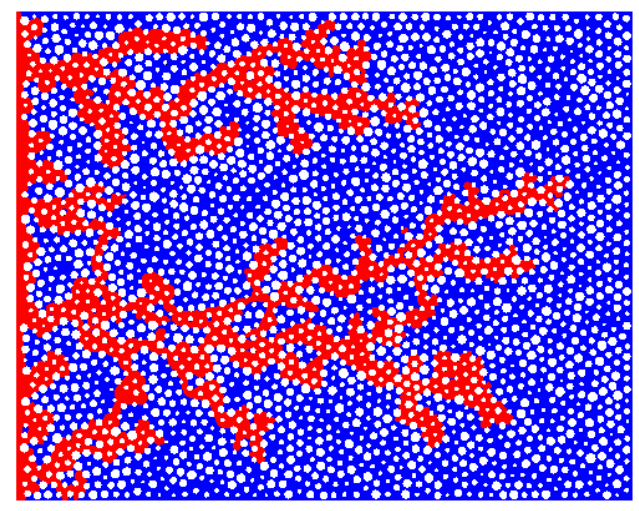

(e) $\left(\phi_{\theta}^{e}, \phi_{0}^{e}\right)+[\partial f \partial t]_{b}$

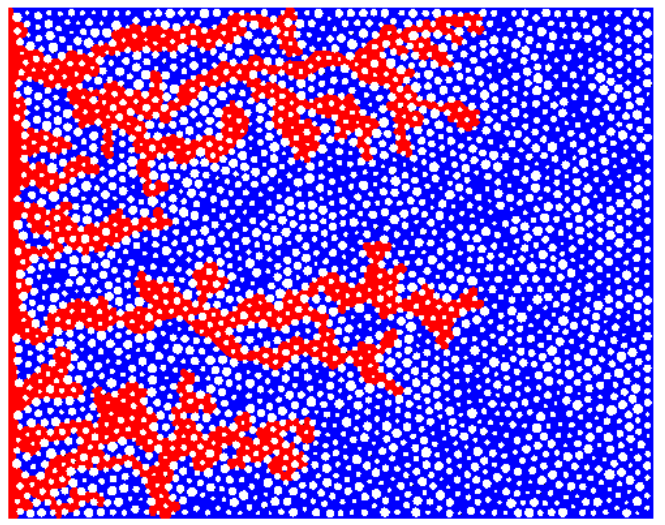

(b) full-physics

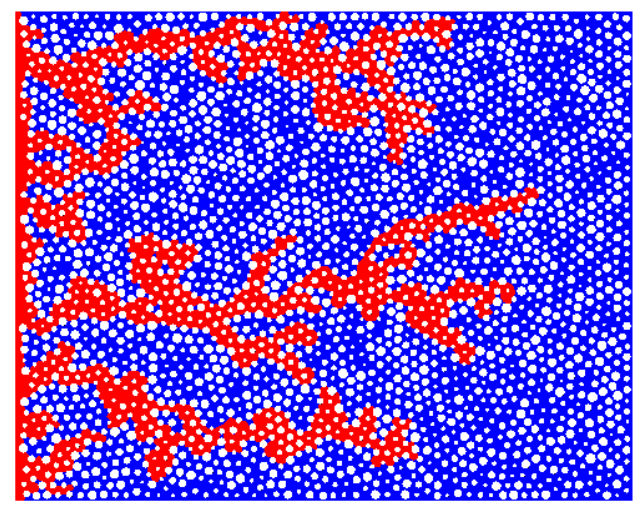

(d) $[\partial / \partial t]_{b+c}$

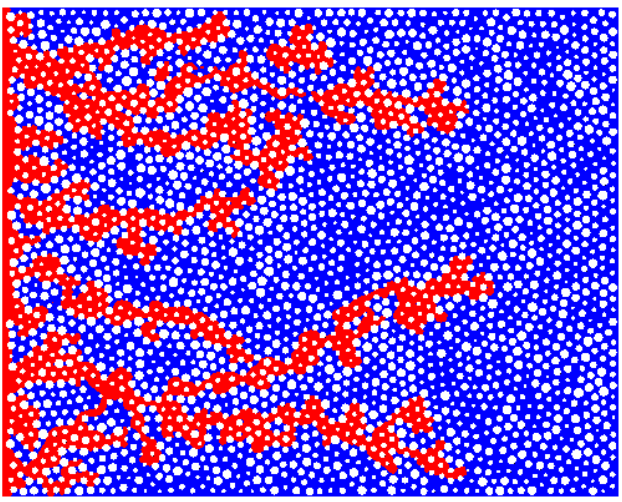

(f) $\not k \nabla \alpha$

Figure 8: Drainage simulations for $M=4 \cdot 10^{-4}$ : (a) fine-scale reference solution, (b) multiphysics solution with full-physics interpolators, ( $-\mathrm{f}$ ) multiphysics solutions with simplified interpolators. (c) no time derivative for basis functions, (d) no time derivative for basis and correction functions, (e) no correction function and no time derivative for basis functions, and (f) no surface tension for correction function. Dual grid has $4 \times 4$ cells. 

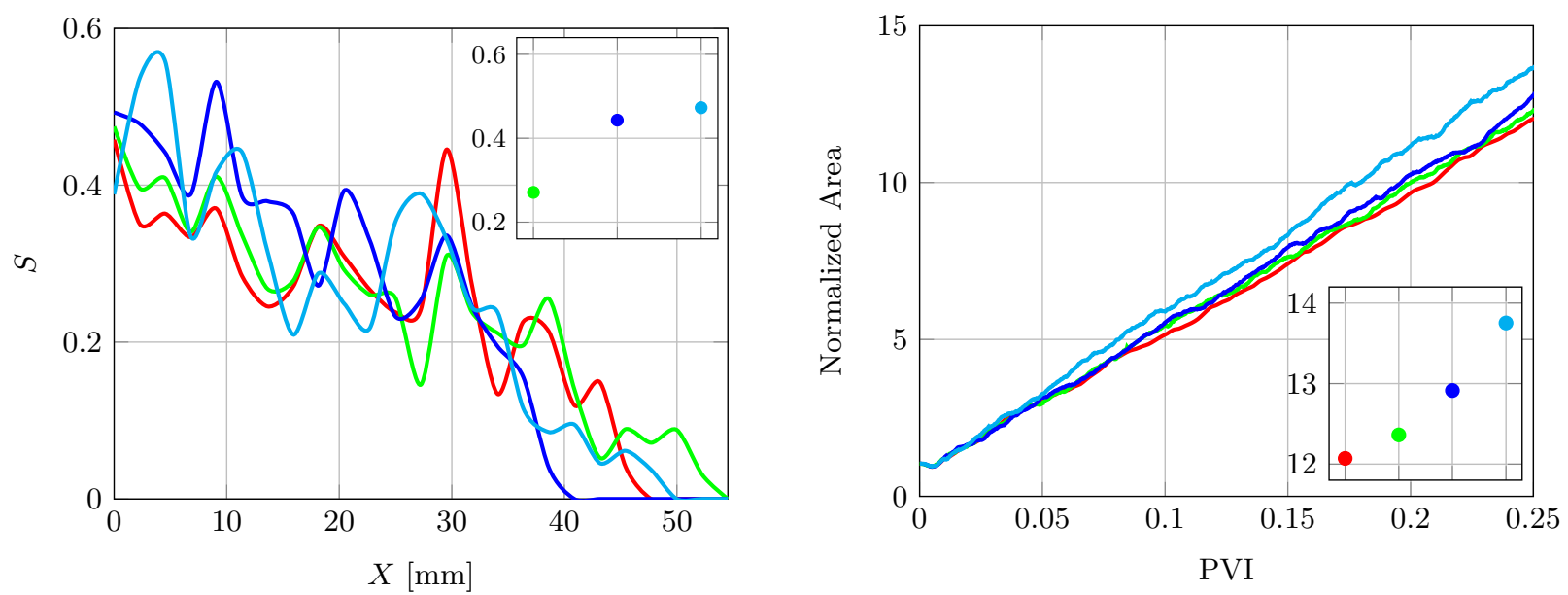

ref $-2 \times 2-4 \times 4-8 \times 8$

Figure 9: Left: average longitudinal profiles of the saturation; the subplot shows the $\mathrm{L}_{2}$-norm difference between the multiphysics and reference curves. Right: fluid-fluid interfacial area (normalized by its value at initial condition) as a function of pore volume injected (PVI) (right); the subplot shows the normalized area values at PVI $=0.25$. Viscosity ratio is $M=4 \cdot 10^{-4}$.

(a) $4 \times 4$

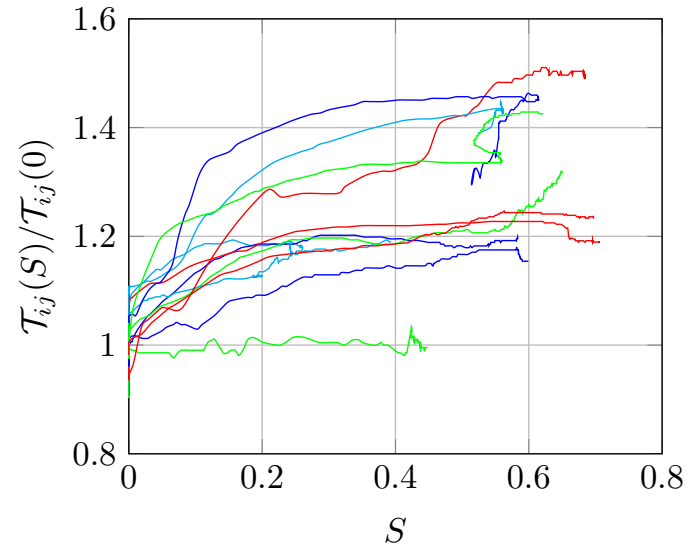

(b) $8 \times 8$

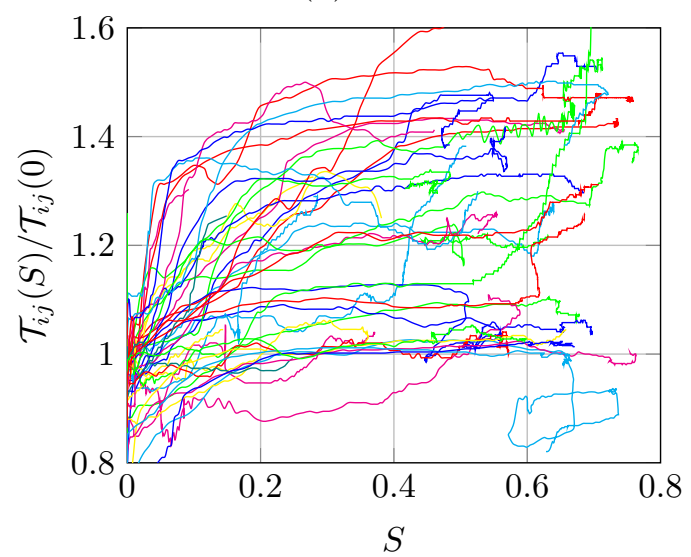

Figure 10: Coarse-scale transmissibilities as functions of the average control-volume saturations for $M=0.2$ and different dual grid sizes. Different lines represent the values for different control volumes (or coarse cells). 
fluid distribution. Since the latter is dictated by the complex interplay of pore-scale forces, it becomes important, in order to obtain accurate transmissibilities, to include the relevant physical processes in the interpolators (basis and correction functions).

In the following we investigate how the quality of the multiphysics solution is affected by transmissibilities computed with an approximate physical description. For the basis functions, the only possible simplification is to neglect the time derivative (see Eq. 13), whereas for the correction functions it is also possible to omit the surface tension term (see Eq. 14).

We fix the dual-grid size to $4 \times 4$ cells and simulate drainage at $M=0.2$. For this test case, the multiphysics algorithm with full-physics interpolators provides a solution in good agreement with the reference (see Figs. 6a,b and 7). However, if the time derivative is neglected in the system that defines the basis functions (Eq. 13 , which then becomes a Stokes-like system of equations), the finger structure becomes more elongated and the front propagates faster (Fig. 6c). This results from an inconsistent description of time relaxation between basis and correction functions. If we omit the time derivative also in the calculation of the correction functions, the penetration length becomes closer to the reference, but a visual comparison of the fluid distribution shows that the fluids pattern remains quite different from the reference (compare Fig. 6a and Fig. 6d). In order to quantify the morphological differences between the invading structures we compare the fluid-fluid interfacial area as a function of the pore volume injected (Fig. 11 a). Neglecting the time derivative in the interpolators leads to an error of about $11 \%$.

Next we investigate the consequences of neglecting the surface tension term. We first omit the correction functions and drop the time derivative in the basis functions, which is equivalent to assume that the velocity relaxation is instantaneous and the interpolators (basis functions) are simply solutions of the Stokes problem. In this case, the multiphysics solution leads to a different front morphology, which is characterized by smaller clusters of defending fluid left behind the front (Fig. 6e), and a systematically larger interfacial area (Fig. 11 ). If we retain the correction functions, but omit the surface tension term (thus accounting for time relaxation), we obtain a similar behavior of the interfacial area (Fig. 11a), indicating that, to obtain accurate fluxes at the control-volume boundaries, it is important to include the surface tension because it has a dominant effect on the front morphology. The reason for the systematically larger interfacial areas is that the approximate descriptions of the physics promote the instability. Indeed, neglecting the time derivatives yields an instantaneous relaxation towards the higher velocity that develops with time as a result of the finger growth, whereas neglecting the surface tension obviously makes viscous forces more dominant by removing the force that opposes finger formation.

If we consider the drainage at low viscosity ratios $\left(M=4 \cdot 10^{-4}\right)$ the errors introduced by the interpolators entailing an incomplete physical description are amplified, particularly in case of an inconsistent description of time relaxation. Indeed, dropping the time derivative in the basis functions leads to snap-off and fingers breaking (Fig. 8f). All multiphysics solutions are unable to capture the correct evolution of the interfacial area and the errors vary between 17 and 25\% (Fig. 11p). This can be critical when surface-controlled processes are involved, such as, e.g., inter-phase reactions.

\section{Conclusions}

Multiphysics algorithms, combining pore-scale and Darcy-scale descriptions, are usually proposed as tools to incorporate pore-scale information in presence of flow regimes for which a fully upscaled description based on Darcy's law breaks down. However, despite the recent advances in pore-scale modeling and high performance computing, it is unrealistic to expect that these techniques can be applicable to problems beyond core or laboratory scale (decimeter or meter scale at most). For large-scale applications the fraction of the domain for which a sub-pore resolution would be computationally tractable is insignificant, and even in presence of infinite computational resources intrinsic practical difficulties would remain due to the lack of information about the pore structure and to the necessity of being able to handle enormous amounts of data. Based on these simple premises it is evident that for practical applications it remains crucial to address the question of the validity of standard macroscopic models and to discuss their possible extensions.

In this context, the Hybrid Multiscale Finite Volume method (Tomin and Lunati, 2013, 2015a b) can serve as a learning tool to assess the validity of the closure assumptions intrinsic to the derivation of 
(a) $M=0.2$

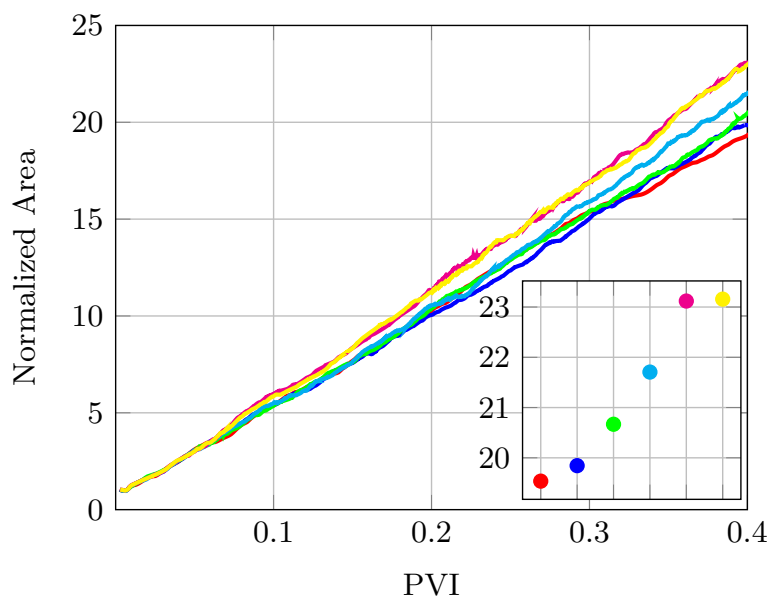

(b) $M=4 \cdot 10^{-4}$

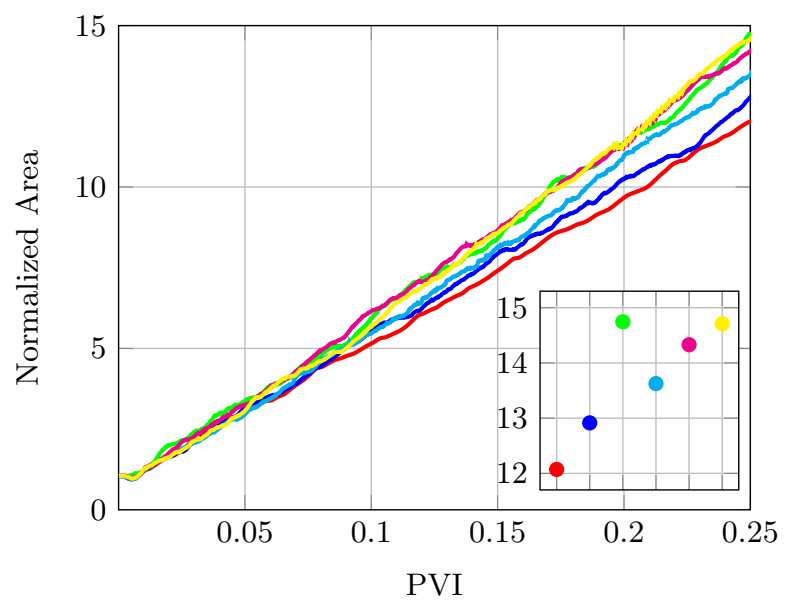

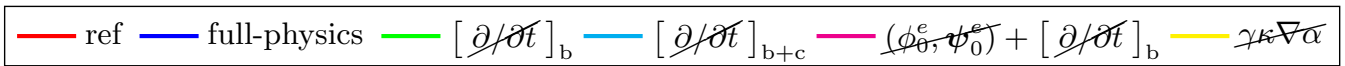

Figure 11: Normalized fluid-fluid interfacial area as a function of pore volume injected (PVI) in case of drainage at $M=0.2$ (left) and $M=4 \cdot 10^{-4}$ (right). The subplots show the area values at PVI $=0.4$ and PVI $=0.25$, respectively. Dual grid has $4 \times 4$ cells.

macromodels and to provide insights into the emergence of the macroscopic equations from the relevant sub-pore mechanisms. Indeed, the accuracy of the multiphysics solution relies on the ability of the global problem, which is constructed by a numerical volume averaging procedure, to correctly transfer information across the control volumes.

In analogy to Darcy's law, the construction of the global-scale problem relies on the assumption of scale separation: the observation length scale (averaging length) has to be large with respect to the typical size characterizing the pore-scale processes and the relaxation time has to be short. Under these conditions, the coarse-scale fluxes are described by a local relationship between the macroscopic fluxes and pressures. As the external pressure forces are almost instantaneously balanced by the drag exerted by the solid matrix, it is not necessary to consider a macroscopic momentum equation.

By means of numerical simulations, we have illustrated that the definition of the scale ratio based on the geometric properties of the porous medium is well justified only for single-phase flow, whereas in case of multiphase flow new characteristic length scales emerge due the coupling between different processes and lead to a dynamic organization of the fluids into large patterns. For moderately unfavorable viscosity ratios $\left(M \sim 10^{-1}\right)$, the multiphysics solution is in very good agreement with the reference. However, in case of strongly unfavorable viscosity ratio $\left(M \sim 10^{-4}\right)$, the accuracy of the multiphysics solution deteriorates owing to the further weakening of scale separation.

In general, the multiphysics solution proved very robust even when the characteristic size of the fluiddistribution patterns is comparable with the observation length. This suggests that macroscopic models based on generalized Darcy's law can provide a satisfactory description of the fluxes also under these circumstances. It has to be noticed, however, that standard generalized Darcy models are based on relative permeabilities that are nonlinear functions of the saturation. The transmissibilities employed in the multiphysics method depend on the actual pore-scale distribution of the fluids, and not only on the average saturation; therefore, they contain much more information than standard relative permeabilities. Even if the macroscopic model would fail, the multiphysics algorithm may be able to better deal with this situation by dynamically computing the coarse-scale parameters. This is illustrated by the fact that the transmissibilities as functions of the average saturation vary greatly from control volume to control volume and from one flow regime to another. Eventually, however, also the multiphysics solution might become inaccurate due to weakening of the scale separation hypothesis. By the comparison between multiphysics and pore-scale 
(reference) solution, we can conclude that the generalized Darcy model is robust only if the relative permeability accounts for the actual morphology of the fluid patterns. This might require constitutive relationships in which the relative permeability is not only a function of the saturation, but also of the dimensionless numbers that characterize the flow regime.

We have also demonstrated that it is important that the Darcy-scale parameters (transmissibilities and right-hand side) are constructed by means of interpolators that take into account the relevant physical processes at the pore scale. (Notice that this is necessary also when a full-physics model is solved for the local problems defined on control volumes and the interpolators only influence the boundary conditions of those problems, which is the case considered here.) Our results indicate that the quality of interpolators and boundary conditions may be crucial in case of unstable regimes. If some relevant physical process is omitted or if the interpolators are not fully consistent (for instance in the way they deal with time relaxation) the multiphysics results might be very inaccurate, owing to the fact that the macro-scale variables do not contain the correct information about the local front dynamics and morphology.

\section{Acknowledgments}

The work was supported by the Swiss National Science Foundation grant PP00P2-144922/1. The authors wish to thank Yves Méheust for help with image analysis and Andrea Ferrari for many constructive discussions.

\section{References}

\section{References}

Aarnes, J.E., Kippe, V., Lie, K.A., Rustad, A.B., 2007. Modelling of multiscale structures in flow simulations for petroleum reservoirs, in: Geometric Modelling, Numerical Simulation, and Optimization. Springer, pp. 307-360.

Aarnes, J.E., Lie, K., Kippe, V., Krogstad, S., 2009. Multiscale methods for subsurface flow, in: Multiscale modeling and simulation in science. Springer, pp. 3-48.

Afkhami, S., Zaleski, S., Bussmann, M., 2009. A mesh-dependent model for applying dynamic contact angles to vof simulations. Journal of Computational Physics 228, 5370-5389.

Alyaev, S., Keilegavlen, E., Nordbotten, J.M., 2014. Analysis of control volume heterogeneous multiscale methods for single phase flow in porous media. Multiscale Model. Simul. 12, 335-363.

Aziz, K., Settari, A., 1979. Petroleum reservoir simulation. Applied Science Publ. Ltd., London, UK.

Battiato, I., Tartakovsky, D.M., Tartakovsky, A.M., Scheibe, T.D., 2011. Hybrid models of reactive transport in porous and fractured media. Advances in Water Resources 34, 1140-1150.

Bear, J., Verruijt, A., 1987. Modeling groundwater flow and pollution. Springer Science \& Business Media.

Bohorquez, P., 2008. Study and numerical simulation of sediment transport in free-surface flow. Ph.D. thesis. University of Malaga.

Bonfigli, G., Jenny, P., 2009. An efficient multi-scale Poisson solver for the incompressible Navier-Stokes equations with immersed boundaries. Journal of Computational Physics 228, 4568-4587.

Darcy, H., 1856. Les Fontaines Publiques de la Ville de Dijon. Dalmont, Paris.

Eckhardt, B., Buehrle, J., 2008. Time-dependent effects in high viscosity fluid dynamics. The European Physical Journal-Special Topics 157, 135-148.

Efendiev, Y., Hou, T.Y., 2008. Multiscale computations for flow and transport in heterogeneous media, in: Quantum Transport. Springer, pp. 169-248.

Efendiev, Y., Hou, T.Y., 2009. Multiscale finite element methods: theory and applications. volume 4. Springer Science \& Business Media.

Ferrari, A., Jimenez-Martinez, J., LeBorgne, T., Méheust, Y., Lunati, I., 2015. Challenges in modeling unstable two-phase flow experiments in micromodels. Water Resources Research 51, 1381-1400.

Ferrari, A., Lunati, I., 2013. Direct numerical simulations of interface dynamics to link capillary pressure and total surface energy. Advances in Water Resources 57, 19-31.

Ferrari, A., Lunati, I., 2014. Inertial effects during irreversible meniscus reconfiguration in angular pores. Advances in Water Resources 74, 1-13.

Hajibeygi, H., Jenny, P., 2009. Multiscale finite-volume method for parabolic problems arising from compressible multiphase flow in porous media. Journal of Computational Physics 228, 5129-5147.

Hajibeygi, H., Karvounis, D., Jenny, P., 2011. A hierarchical fracture model for the iterative multiscale finite volume method. Journal of Computational Physics 230, 8729-8743.

Hajibeygi, H., Lee, S.H., Lunati, I., 2012. Accurate and efficient simulation of multiphase flow in a heterogeneous reservoir with error estimate and control in the multiscale finite-volume framework. SPE Journal 17, 1071-1083. 
Hajibeygi, H., Tchelepi, H.A., 2014. Compositional multiscale finite-volume formulation. SPE-163664-PA, SPE Journal 19, 316-326.

Helmig, R., 1997. Multiphase flow and transport processes in the subsurface: a contribution to the modeling of hydrosystems. Springer-Verlag, Berlin.

Hirt, C.W., Nichols, B.D., 1981. Volume of fluid (VOF) method for the dynamics of free boundaries. Journal of Computational Physics 39, 201-225.

Holzbecher, E.O., 1998. Modeling density-driven flow in porous media: principles, numerics, software. Springer Science \& Business Media.

Jenny, P., Lee, S.H., Tchelepi, H.A., 2003. Multi-scale finite-volume method for elliptic problems in subsurface flow simulation. Journal of Computational Physics 187, 47-67.

455 Jenny, P., Lunati, I., 2009. Modeling complex wells with the multi-scale finite-volume method. Journal of Computational Physics 228, 687-702.

Kneafsey, T.J., Pruess, K., 2010. Laboratory flow experiments for visualizing carbon dioxide-induced, density-driven brine convection. Transport in porous media 82, 123-139.

Künze, R., Lunati, I., 2012. An adaptive multiscale method for density-driven instabilities. Journal of Computational Physics $231,5557-5570$

Künze, R., Tomin, P., Lunati, I., 2014. Local modeling of instability onset for global finger evolution. Advances in Water Resources 70, 148-159.

Lafaurie, B., Nardone, C., Scardovelli, R., Zaleski, S., Zanetti, G., 1994. Modelling merging and fragmentation in multiphase flows with SURFER. Journal of Computational Physics 113, 134-147.

Lee, S.H., Wolfsteiner, C., Tchelepi, H.A., 2008. Multiscale finite-volume formulation for multiphase flow in porous media: black oil formulation of compressible, three-phase flow with gravity. Computational Geosciences 12, 351-366.

Lenormand, R., Touboul, E., Zarcone, C., 1988. Numerical models and experiments on immiscible displacements in porous media. Journal of Fluid Mechanics 189, 165-187.

Løvoll, G., Méheust, Y., Toussaint, R., Schmittbuhl, J., Måløy, K.J., 2004. Growth activity during fingering in a porous Hele-Shaw cell. Physical Review E 70, 026301.

Lunati, I., Attinger, S., Kinzelbach, W., 2002. Macrodispersivity for transport in arbitrary non-uniform flow fields: Asymptotic and pre-asymptotic results. Water resources research 38, 1187.

Lunati, I., Jenny, P., 2006. Multi-scale finite-volume method for compressible flow in porous media. Journal of Computational Physics 216, 616-636.

475 Lunati, I., Jenny, P., 2008. Multiscale finite-volume method for density-driven flow in porous media. Computational Geosciences $12,337-350$

Lunati, I., Lee, S., 2009. An operator formulation of the multiscale finite-volume method with correction function. Multiscale Modeling and Simulation 8, 96-109.

Lunati, I., Or, D., 2009. Gravity-driven slug motion in capillary tubes. Physics of Fluids (1994-present) $21,052003$.

480 Lunati, I., Tyagi, M., Lee, S.H., 2011. An iterative multiscale finite volume algorithm converging to the exact solution. Journal of Computational Physics 230, 1849-1864.

Méheust, Y., Løvoll, G., Måløy, K.J., Schmittbuhl, J., 2002. Interface scaling in a two-dimensional porous medium under combined viscous, gravity, and capillary effects. Physical Review E 66, 051603.

Molins, S., Trebotich, D., Yang, L., Ajo-Franklin, J.B., Ligocki, T.J., Shen, C., Steefel, C.I., 2014. Pore-scale controls on calcite dissolution rates from flow-through laboratory and numerical experiments. Environmental science \& technology 48 , $7453-7460$.

Møyner, O., Lie, K.A., 2014. A multiscale two-point flux-approximation method. Journal of Computational Physics 275, $273-293$.

OpenFOAM, 2012. The Open Source CFD Toolbox: User Guide. OpenFOAM Foundation, version 2.1.1.

Saffman, P.G., Taylor, G., 1958. The penetration of a fluid into a porous medium or Hele-Shaw cell containing a more viscous liquid, in: Proceedings of the Royal Society of London A: Mathematical, Physical and Engineering Sciences, The Royal Society. pp. 312-329.

Sandve, T.H., Keilegavlen, E., Nordbotten, J.M., 2014. Physics-based preconditioners for flow in fractured porous media. Water Resources Research 50, 1357-1373.

495 Scardovelli, R., Zaleski, S., 1999. Direct numerical simulation of free-surface and interfacial flow. Annual Review of Fluid Mechanics 31, 567-603.

Scheibe, T.D., Murphy, E.M., Chen, X., Rice, A.K., Carroll, K.C., Palmer, B.J., Tartakovsky, A.M., Battiato, I., Wood, B.D., 2015a. An analysis platform for multiscale hydrogeologic modeling with emphasis on hybrid multiscale methods. Groundwater 53, 38-56.

500 Scheibe, T.D., Perkins, W.A., Richmond, M.C., McKinley, M.I., Romero-Gomez, P.D., Oostrom, M., Wietsma, T.W., Serkowski, J.A., Zachara, J.M., 2015b. Pore-scale and multiscale numerical simulation of flow and transport in a laboratoryscale column. Water Resources Research .

Tartakovsky, A.M., Tartakovsky, D.M., Scheibe, T.D., Meakin, P., 2008. Hybrid simulations of reaction-diffusion systems in porous media. SIAM Journal on Scientific Computing 30, 2799-2816.

505 Tomin, P., Lunati, I., 2013. Hybrid multiscale finite volume method for two-phase flow in porous media. Journal of Computational Physics 250, 293-307.

Tomin, P., Lunati, I., 2015a. Local-global splitting for spatiotemporal-adaptive multiscale methods. Journal of Computational Physics 280, 214-231.

Tomin, P., Lunati, I., 2015b. Spatiotemporal adaptive multiphysics simulations of drainage-imbibition cycles. Computational 
Geosciences , 1-14.

Tyukhova, A.R., Kinzelbach, W., Willmann, M., 2015. Delineation of connectivity structures in 2-d heterogeneous hydraulic conductivity fields. Water Resources Research 51, 5846-5854.

Vinningland, J.L., Johnsen, Ø., Flekkøy, E.G., Toussaint, R., Måløy, K.J., 2007. Experiments and simulations of a gravitational granular flow instability. Physical Review E 76, 051306.

Wolfsteiner, C., Lee, S.H., Tchelepi, H.A., 2006. Well modeling in the multiscale finite volume method for subsurface flow simulation. Multiscale Modeling and Simulation 5, 900-917.

Yang, X., Scheibe, T.D., Richmond, M.C., Perkins, W.A., Vogt, S.J., Codd, S.L., Seymour, J.D., McKinley, M.I., 2013. Direct numerical simulation of pore-scale flow in a bead pack: Comparison with magnetic resonance imaging observations. Advances in Water Resources 54, 228-241. 
Scales of Multiphase Flow

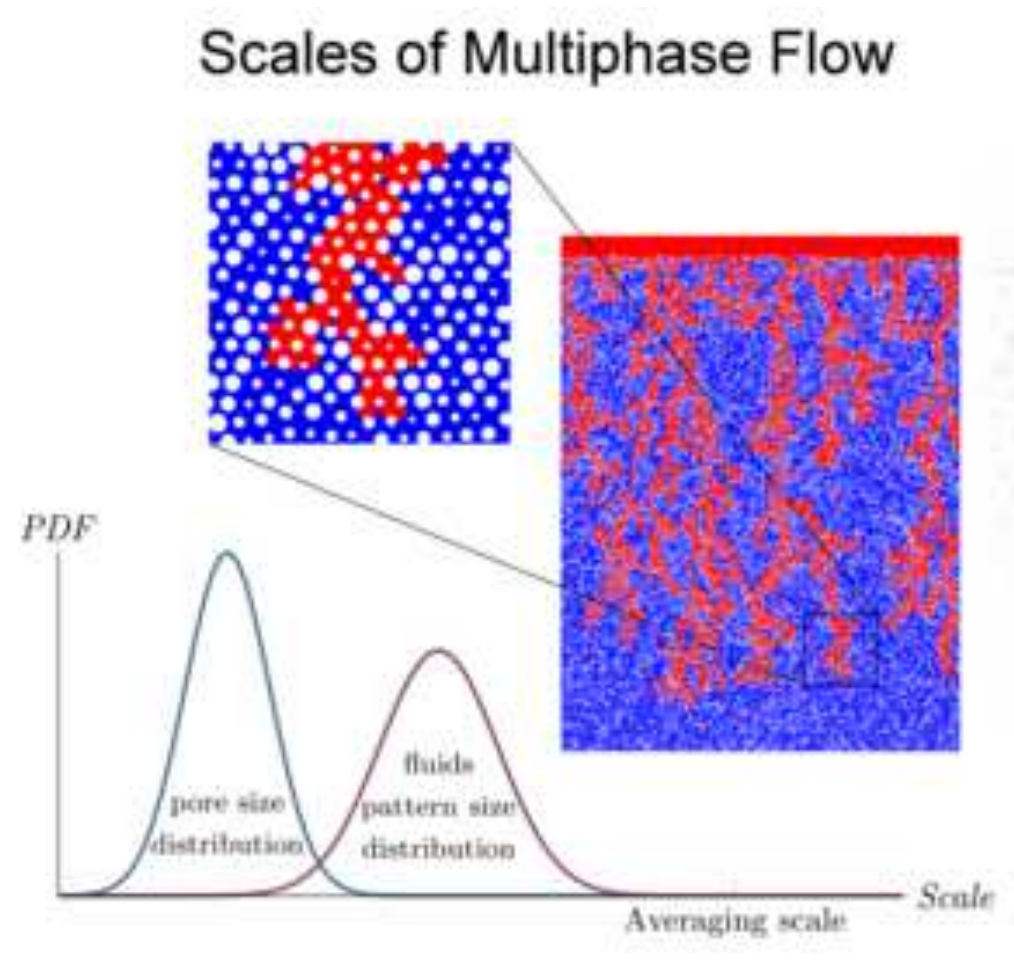

Numerical Averaging by Multiphysics Algorithm
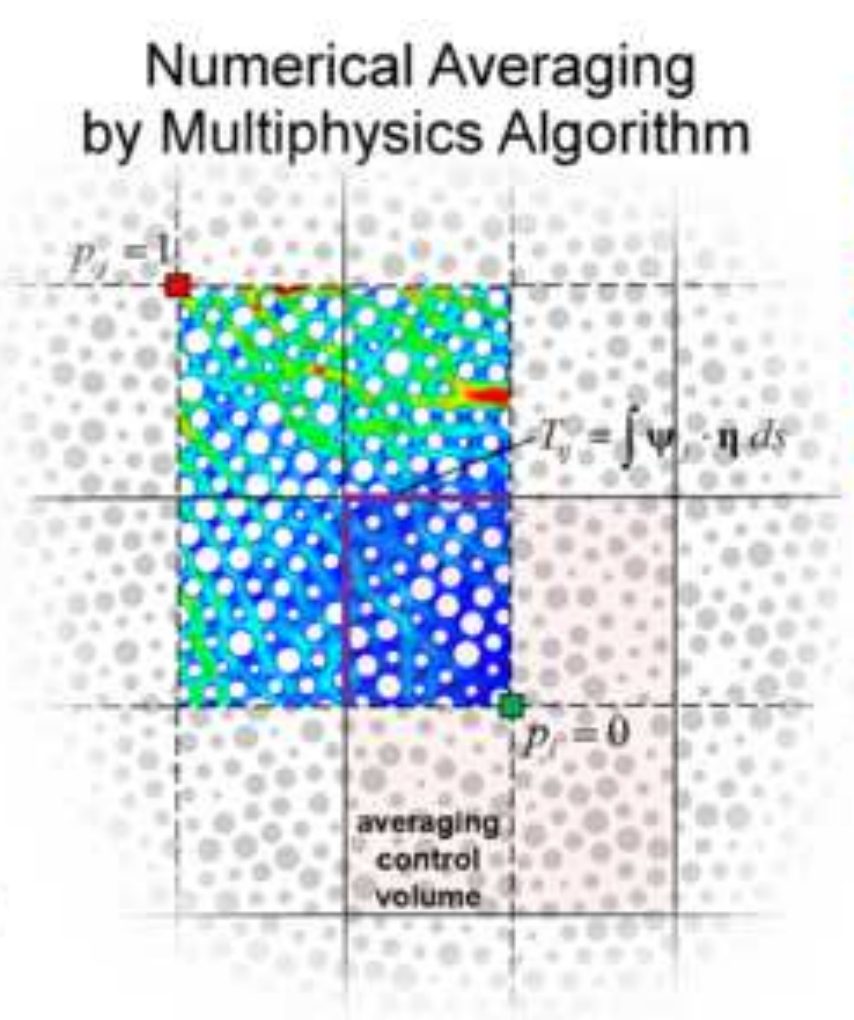

Pore-Scale Physics
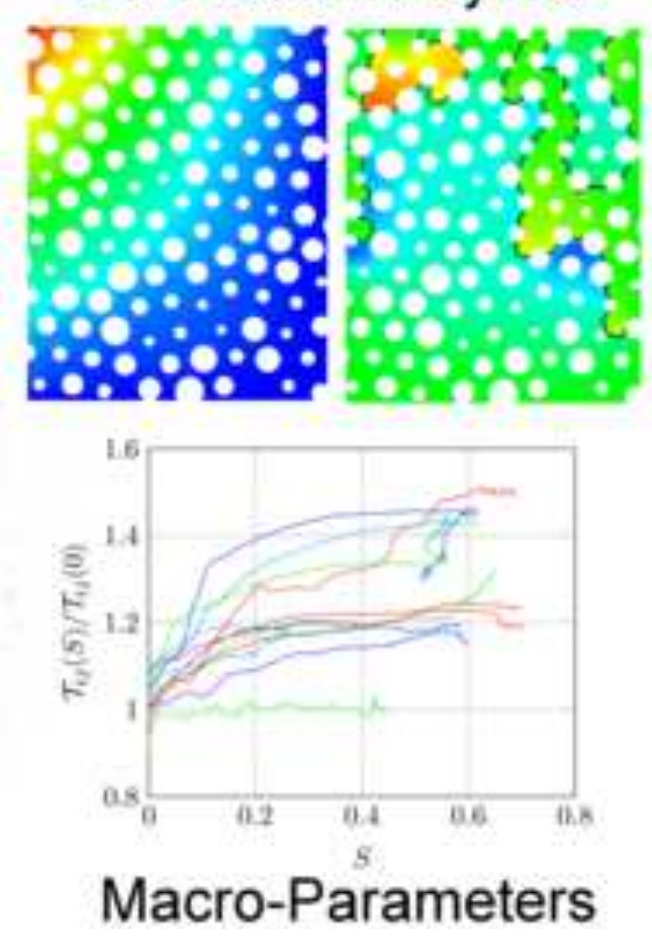